\title{
Integrated analysis of long non-coding RNA competing interactions revealed potential biomarkers in cervical cancer: Based on a public database
}

\author{
WEN-JUAN WU ${ }^{1}$, YANG SHEN ${ }^{2}$, JING SUI ${ }^{1}$, CHENG-YUN LI ${ }^{1}$, SHENG YANG ${ }^{1}$, \\ SI-YI XU ${ }^{1}$, MAN ZHANG ${ }^{3}$, LI-HONG YIN ${ }^{1}$, YUE-PU PU ${ }^{1}$ and GE-YU LIANG ${ }^{1}$ \\ ${ }^{1}$ Key Laboratory of Environmental Medicine Engineering, Ministry of Education, \\ School of Public Health, Southeast University; ${ }^{2}$ Department of Obstetrics and Gynecology, \\ Zhongda Hospital, School of Medicine, Southeast University; ${ }^{3}$ Department of Medical Insurance, \\ School of Public Health, Southeast University, Nanjing, Jiangsu 210009, P.R. China
}

Received September 21, 2017; Accepted March 21, 2018

DOI: $10.3892 / \mathrm{mmr} .2018 .8846$

\begin{abstract}
Cervical cancer (CC) is a common gynecological malignancy in women worldwide. Using an RNA sequencing profile from The Cancer Genome Atlas (TCGA) and the CC patient information, the aim of the present study was to identify potential long non-coding RNA (lncRNA) biomarkers of $\mathrm{CC}$ using bioinformatics analysis and building a competing endogenous RNA (ceRNA) co-expression network. Results indicated several CC-specific IncRNAs, which were associated with CC clinical information and selected some of them for validation and evaluated their diagnostic values. Bioinformatics analysis identified $51 \mathrm{CC}$-specific lncRNAs (fold-change $>2$ and $\mathrm{P}<0.05$ ), and 42 of these were included in ceRNA network consisting of lncRNA-miRNA-mRNA interactions. Further analyses revealed that differential expression levels of 19 IncRNAs were significantly associated with different clinical features $(\mathrm{P}<0.05)$. A total of 11 key lncRNAs in the ceRNA network for reverse transcription-quantitative polymerase chain reaction (RT-qPCR) analysis to detect their expression levels in 31 pairs of CC clinical samples. The results indicated that 7 lncRNAs were upregulated and 4 lncRNAs were downregulated in CC patients. The fold-changes between the RT-qPCR experiments and the TCGA bioinformatics analyses were the same. Furthermore, the area under the receiver operating characteristic (ROC) curve of four IncRNAs (EMX20S, MEG3, SYS1-DBNDD2 and MIR9-3HG) indicated that their combined use may have
\end{abstract}

Correspondence to: Professor Ge-Yu Liang, Key Laboratory of Environmental Medicine Engineering, Ministry of Education, School of Public Health, Southeast University, 87 Dingjiaqiao Street, Nanjing, Jiangsu 210009, P.R. China

E-mail: lianggeyu@163.com

Key words: long non-coding RNAs, cervical cancer, ceRNA network, TCGA, biomarkers a significant diagnostic value in $\mathrm{CC}(\mathrm{P}<0.05)$. To the best of our knowledge, the present study is the first to have identified CC-specific lncRNAs to construct a ceRNA network and has also provided new insights for further investigation of a lncRNA-associated ceRNA network in CC. In additon, the verification results suggested that the method of bioinformatics analysis and screening of lncRNAs was accurate and reliable. To conclude, the use of multiple lncRNAs may thus improve diagnostic efficacy in CC. In addition, these specific lncRNAs may serve as new candidate biomarkers for clinical diagnosis, classification and prognosis of CC.

\section{Introduction}

Cervical cancer (CC) is one of the most lethal cancers with increasing incidence and mortality over the past decades, and is the second most common female malignant disease worilwide (1). According to the latest world cancer statistics, approximately 529,800 female are diagnosed with CC and approximately 275,100 die worldwide each year, making CC the second fastest growing cancer and a serious threat to women's health (2). Meanwhile, the age of CC incidence has progressively decreased, which has attracted wide attention. Recent studies have shown that lifestyle, environmental pollution, population aging genetic predisposition, HPV infection and the impact of hormones are the important causes of CC (3). Although the morbidity and mortality of $\mathrm{CC}$ has declined in the past 30 years, the 5-year survival rate of advanced-stage patients still below $40 \%$ (4). Therefore, in order to improve the cure percentage of $\mathrm{CC}$, it is important to understand its molecular mechanism and identify effective diagnostic and prognostic biomarkers.

Long non-coding RNA (lncRNA) is a non-coding RNA more than 200 nucleotides in length (5). More and more evidence has showed that lncRNAs is an important part of a complex gene regulatory network which regulates gene expression at the epigenetics and transcriptome levels (6). The lncRNAs are differently expressed in many kinds of cancers $(7,8)$, including gastric, lung and ovarian cancer (9-11). In addition, abnormal expression of lncRNAs has been related 
to metastasis, recurrence, and prognosis of various human tumors (12). More importantly, Compared with protein coding mRNAs and miRNA, IncRNAs have greater tissue specificity (13). Thus, discovery of differentially expressed lncRNAs in CC may be important for the diagnosis and the identifications for this disease.

Recently, the hypothesis of competing endogenous RNAs (ceRNAs) has suggested that RNA transcripts interact via miRNA response elements. Increasing evidences indicates that lncRNAs, mRNAs and pseudogene acting as ceRNAs can be regulated by MREs and play key funtions in metastasis, tumorigenesis and progression of tumors (14). Meanwhile, ceRNA activity also plays an important roles in the transcriptome and increasing evidence has shown that genetic information is closely related to pathological change in most cancers (15).

HPV infection alone is not be the only factor CC formation. Host genetic variations also play an important roles in the development of CC (16). With the development of high-throughput gene sequencing technologies and molecular biology methods, we can use these new tools for the discovery and identification of cancer biomarkers $(17,18)$. However, studies to date have lacked the integrated analysis of large samples and the sensitivity of CC-specific IncRNAs biomarkers. In addition, small sample studies do not have the statistical power to explain the relationships between abnormal IncRNAs and CC patients' clinical features Recently, The Cancer Genome Atlas (TCGA) (http://cancergenome.nih.gov) database has collected and provided a large sample size of CC genome sequencing data. The aim of our study was to solve the problem of small sample size and improve the accuracy and reliability of results by using TCGA RNA sequencing data from CC patients to find CC-related IncRNAs. In this study, we collected whole transcriptome RNA sequencing data of $307 \mathrm{CC}$ tissues specimens and six adjacent nontumor tissue specimens through the TCGA database. To the best of our knowledge, our study is the first time to investigate the CC-related lncRNA expression profiles through the use of a large-scale samples RNA sequencing database. Subsequently, the reverse transcription-quantitative polymerase chain reaction (RT-qPCR) was used to validate part of the bioinformatics analysis results by 31 pairs of newly diagnosed CC clinical samples. This new method of finding CC-related lncRNAs through the ues of ceRNA network can help determine the potential functions of lncRNAs in CC progression and development.

\section{Materials and methods}

Patients and samples. Following the TCGA guidelines, we downloaded RNA sequencing data and clinical pathological information from 307 cases of cervical squamous cell carcinoma (CESC) in the TCGA database (up to Decenber 1, 2016). Then, we excluded cases without completed analysis data, with a histologic diagnosis that was not CESC, with more than two malignant tumors, and those which had received preoperative chemoradiation. Finally, 289 CC patients remained for analysis based on the above exclusion criteria. From these patients, RNA sequencing data from 289 tumor tissues and six nontumor tissues were obtained. Using the international Federation of Gynecology and Obstetrics (FIGO) staging system, we divided the patients into three groups, FIGO stage I were 158 patients, FIGO stage II, 68 patients; and FIGO stage III-IV, 63 patients.

In addition, 31 tissue specimens (tumor tissues and adjacent normal tissue) were collected between 2016 and 2017 at the Zhongda Hospital of Southeast University (Nanjing, China) form CC patients, aged 23-64 years for RT-qPCR analysis. Tissues specimens were rapidly frozen in RNAlater (Ambion; Thermo Fisher Scientific, Inc., Austin, TX, USA) and were stored in liquid nitrogen for subsequent RNA extraction and RT-qPCR analysis. These 31 patients were diagnosed of CC based on the histopathology and clinical history. All patients signed informed consent, and this study also was approved by the ethics committee of Zhongda Hospital Southeast University.

RNA sequence data collects and analysis. The CESC-RNA sequencing data (level 3) and clinical information were downloaded from TCGA database until December 1, 2016. The TCGA database provides normalized count data for RNA sequencing through the RNASeqV2 system, which contained the IncRNA and mRNA sequencing data. Meanwhile, CESC miRNA sequencing data also were obtained through the TCGA database. Level 3 miRNA sequencing base data were obtained through Illumina HiSeq 2000 miRNA sequencing platforms (Illumina, Inc., San Diego, CA, USA). The RNA sequencing data from these CESC patients tissues specimens had previously been normalized to the TCGA database. We then further analyzed the differentially expressed RNA sequencing data by bioinformatics analysis. The bioinformatics analysis is shown in Fig. 1.

Functional enrichment of Gene Ontology (GO) and pathway analysis. We analyzed the biological processes of aberrantly expressed intersection mRNAs through the Database for Annotation, Visualization, and Integrated Discovery (DAVID) (http://david.abcc.ncifcrf.gov/), which used GO database to investigate the potential functions of these aberrantly expressed intersection mRNAs (19). The potential functions of mRNAs participating in the pathways were then analyzed using the Kyoto Encyclopedia of Genes and Genomes (KEGG) database.

Construction of ceRNA network. We made use of the theory in which IncRNAs regulate miRNA by binding and sequestering them and miRNAs in turn regulate mRNAs via IncRNA-miRNA-mRNA interactions in the competitive endogenous RNA network (20). Therefore, we selected the abnormally expressed $\operatorname{lncRNA}$, miRNA, andmRNA in the intersection of three groups based on fold-change $>2.0$ and $\mathrm{P}<0.05$. Next, we used miRanda (http://www.microrna.org) to predict the miRNA targets and investigate lncRNA-miRNA relationships. Meanwhile, Target scan (http://www.targetscan.org/) and miRbase targets (http://mirdb.org) were used to predict miRNA target genes. Finally, we combined the differentially expressed data from TCGA with the predicted targets of miRNAs to select and the results of miRNAs that predicted target lncRNAs and mRNAs to select commonly regulated lncRNAs and mRNAs. In accordance with the principle of negative regulation of ceRNA, we select the most negative regulated miRNA, IncRNAs and mRNA to build the ceRNA 


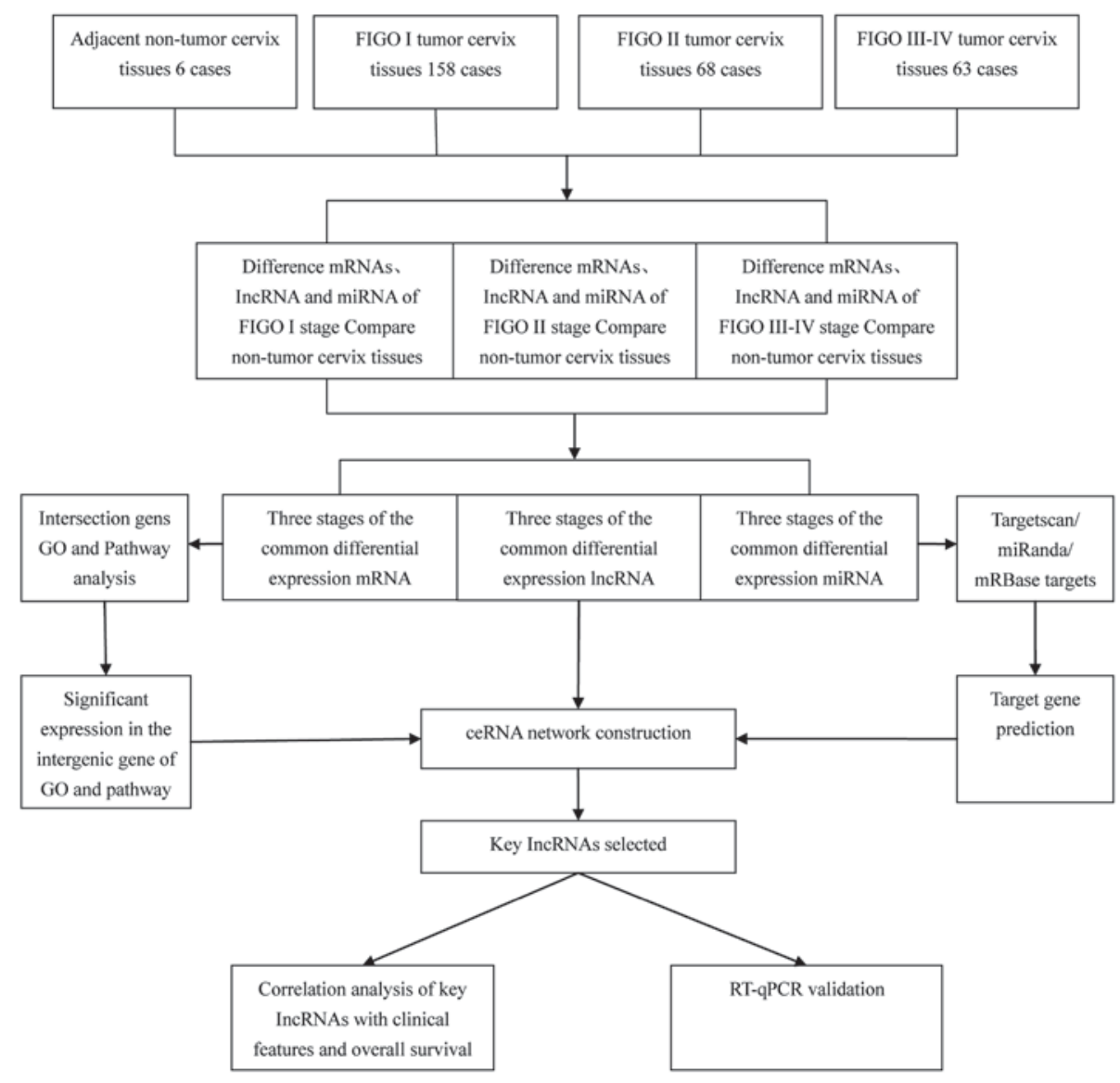

Figure 1. Flow chart of bioinformatics analysis and ceRNA network analysis. ceRNA, competing endogenous RNA; FIGO, International Federation of Gynecology and Obstetrics; GO, Gene Ontology; RT-qPCR, reverse transcription-quantitative polymerase chain reaction; IncRNA, long non-coding RNA.

regulatory network, using Cytoscape version 3.0 to construct it (21). Fig. 1 shows a flow chart outlining the steps used to bulid the ceRNA network.

Association analysis between CC specific IncRNAs and clinical features. We chose the key lncRNAs to be included in the ceRNA network according to the comprehensively bioinformatics analysis of the CC RNA sequencing data in TCGA. In the next step, we further analyzed the relationships between CC-specific lncRNAs and patients clinical features including race, pathological stage, tumor grade, TNM stage, FIGO stage and HPV infection. Subsequently, we chose several of the key IncRNAs in the ceRNA network and validate the accuracy and reliability of results from the bioinformatics analysis using RT-qPCR to analyze 31 newly diagnosed CC patients.

Extraction of total RNA from clinical samples and $R T-q P C R$ verification of bioinformatics results. We random selected 17 key lncRNAs associated with CC patients clinical features that had high association scores in the above bioinformatics ceRNA network. Then, we utilized RT-qPCR to analyzed the actual expression levels of these lncRNAs in 31 newly diagnosed CC patients. We chose GAPDH as the endogenous standard to confirm the accuracy and reliability of our bioinformatics analysis. Total RNA were isolated from tissues specimens of the CC patients using TRIzol reagent (Invitrogen; Thermo Fisher Scientific, Inc., Waltham, MA, USA) according to the manufacturer's protocol, and the purity of the isolated RNA was assessed using NanoDrop 2000 spectrometer (Thermo Fisher Scientific, Inc.). Reverse transcription reactions and RT-qPCR were performed according to the manufacturer's protocol, using the reverse transcription system and qPCR Master Mix kit (Promega Corporation, Madison, WI, USA) as well as the Step One Plus ${ }^{\mathrm{TM}}$ PCR System (Applied Biosystems; Thermo Fisher Scientific, Inc.) to detect the expression levels of lncRNAs. All the primers were produced by Generay Biotech Co., Ltd. (Shanghai, China). The RT-qPCR results were calculated using the $2^{-\Delta \Delta C q}$ method (22) with the formula $\left[\Delta \mathrm{Cq}=\left(\mathrm{Cq}_{\mathrm{RNAs}}-\mathrm{Cq}_{\mathrm{GAPDH}}\right)\right.$ and $\left.\Delta \Delta \mathrm{Cq}=\Delta \mathrm{Cq}_{\text {tumor tissues }}-\Delta \mathrm{Cq}_{\text {adjacent non-tumor tissues }}\right]$.

Statistical analysis. Data analysis was performed using SPSS software version 24.0 (IBM Corp., Armonk, NY, USA). The final results were expressed as mean \pm standard deviation. Student's t-test were used to compare the fold-change between groups of sequencing data. In all cases, $\mathrm{P}<0.05$ was considered to indicate a statistically significant difference. In addition, we used receiver operating characteristic (ROC) curves and the area under the curve (AUC) to judge the diagnostic value of 6 lncRNAs in CC patients.

\section{Results}

Cancer specific lncRNAs in CC. Base on TCGA database 'Level 3' CESC RNA-Sequencing (RNA-Seq) data, we 
Table I. Differentially expressed intersection lncRNAs between FIGO stage I/Normal, FIGO stage II/Normal and FIGO stage III-IV/Normal.

\begin{tabular}{|c|c|c|c|c|}
\hline Name (lncRNA) & Gene ID & Regulation & Average fold-change & $-\log (\mathrm{P})$ \\
\hline EMX2OS & 196047 & Down & -81.30 & 5.921 \\
\hline MIR4697HG & 283174 & Down & -24.39 & 4.096 \\
\hline MIR100HG & 399959 & Down & -20.00 & 6.778 \\
\hline MBNL1-AS1 & 401093 & Down & -14.78 & 4.075 \\
\hline MEG3 & 55384 & Down & -9.46 & 3.989 \\
\hline LINC01140 & 339524 & Down & -9.38 & 3.550 \\
\hline A2M-AS1 & 144571 & Down & -9.09 & 3.509 \\
\hline TPTEP1 & 387590 & Down & -8.33 & 4.281 \\
\hline NR2F1-AS1 & 441094 & Down & -8.11 & 3.611 \\
\hline MIR99AHG & 388815 & Down & -7.89 & 3.605 \\
\hline LINC00341 & 161176 & Down & -7.14 & 4.617 \\
\hline SMIM10L2B & 644596 & Down & -6.00 & 6.015 \\
\hline LINC00663 & 284440 & Down & -5.08 & 3.868 \\
\hline EPB41L4A-AS1 & 114915 & Down & -5.00 & 4.382 \\
\hline LINC00312 & 29931 & Down & -5.00 & 5.436 \\
\hline LINC00950 & 92973 & Down & -4.11 & 3.353 \\
\hline SYS1-DBNDD2 & 767557 & Down & -3.85 & 3.970 \\
\hline SNHG7 & 84973 & Down & -3.75 & 7.000 \\
\hline ATP1A1-AS1 & 84852 & Down & -3.66 & 3.732 \\
\hline RASA4CP & 401331 & Down & -3.66 & 3.974 \\
\hline ILF3-AS1 & 147727 & Down & -3.61 & 3.879 \\
\hline INE2 & 8551 & Down & -3.61 & 5.543 \\
\hline FLJ10038 & 55056 & Down & -3.37 & 6.436 \\
\hline ACVR2B-AS1 & 100128640 & Down & -3.37 & 3.619 \\
\hline FAM66C & 440078 & Down & -3.37 & 3.522 \\
\hline AMZ2P1 & 201283 & Down & -3.37 & 3.508 \\
\hline LOH12CR2 & 503693 & Down & -3.33 & 3.032 \\
\hline ZNF876P & 642280 & Down & -3.06 & 5.301 \\
\hline FTX & 100302692 & Down & 2.40 & 4.494 \\
\hline MIR9-3HG & 254559 & Up & 47.43 & 2.974 \\
\hline TMPO-AS1 & 100128191 & $\mathrm{Up}$ & 7.15 & 4.641 \\
\hline GOLGA2P5 & 55592 & Up & 5.93 & 6.699 \\
\hline CDKN2B-AS1 & 100048912 & $\mathrm{Up}$ & 5.85 & 3.931 \\
\hline MST1P2 & 11209 & Up & 5.49 & 3.832 \\
\hline LINC00467 & 84791 & Up & 5.39 & 3.802 \\
\hline DDX12P & 440081 & Up & 5.31 & 3.102 \\
\hline ASMTL-AS1 & 80161 & Up & 4.92 & 3.468 \\
\hline GEMIN8P4 & 492303 & Up & 4.72 & 3.610 \\
\hline GOLGA2P10 & 80154 & Up & 4.55 & 4.017 \\
\hline OIP5-AS1 & 729082 & Up & 3.09 & 3.046 \\
\hline LOC 146880 & 146880 & Up & 2.59 & 4.999 \\
\hline EP400NL & 347918 & $\mathrm{Up}$ & 2.33 & 7.000 \\
\hline
\end{tabular}

A total of 42 CC specific lncRNAs for competing endogenous RNA network construction with absolute fold-change $>2.0$, P<0.05. Normal represents adjacent non-tumor cervical tissues. lncRNA, long non-coding RNA; FIGO stage, The International Federation of Gynecology and Obstetrics staging.

observed that 71 lncRNAs were abnormality expressed in $289 \mathrm{CC}$ patients tumor tissues compared to 6 adjacent normal cervical tissues with a fold-change $>2$ and $\mathrm{P}<0.05$.
Subsequently, we obtained abnormally expressed lncRNAs from 68 FIGO stage I CC tissues, 68 FIGO stage II tissues, and 71 FIGO stage III-IV tissues when compared to adjacent 
A Stage I/normal Stage II/normal

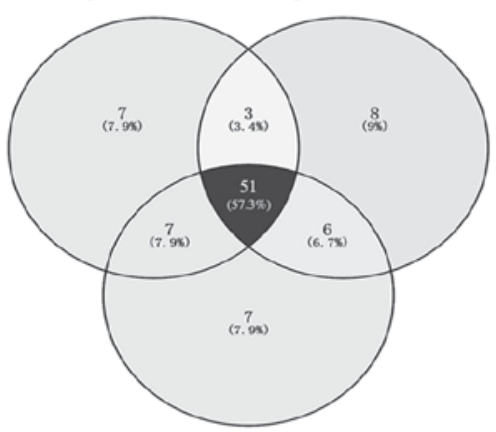

Stage III-IV/normal

IncRNA
B Stage I/normal Stage II/normal

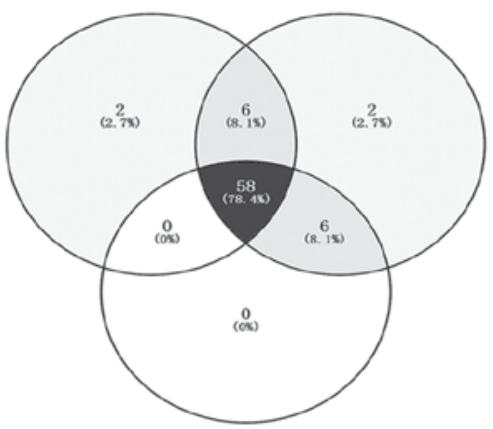

Stage III-IV/normal

miRNA
C Stage I/normal Stage II/normal

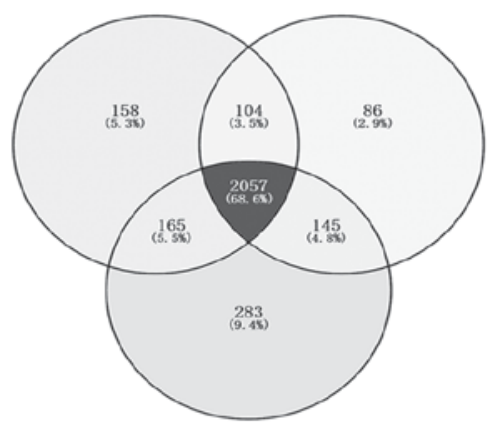

Stage III-IV/normal

mRNA

Figure 2. Venn diagram analysis of differentially expressed (A) lncRNAs, (B) mRNAs and (C) miRNAs between FIGO stage I/Normal, FIGO stage II/Normal, FIGO stage III-IV/Normal. lncRNA, long non-coding RNA; FIGO stage, The International Federation of Gynecology and Obstetrics staging.
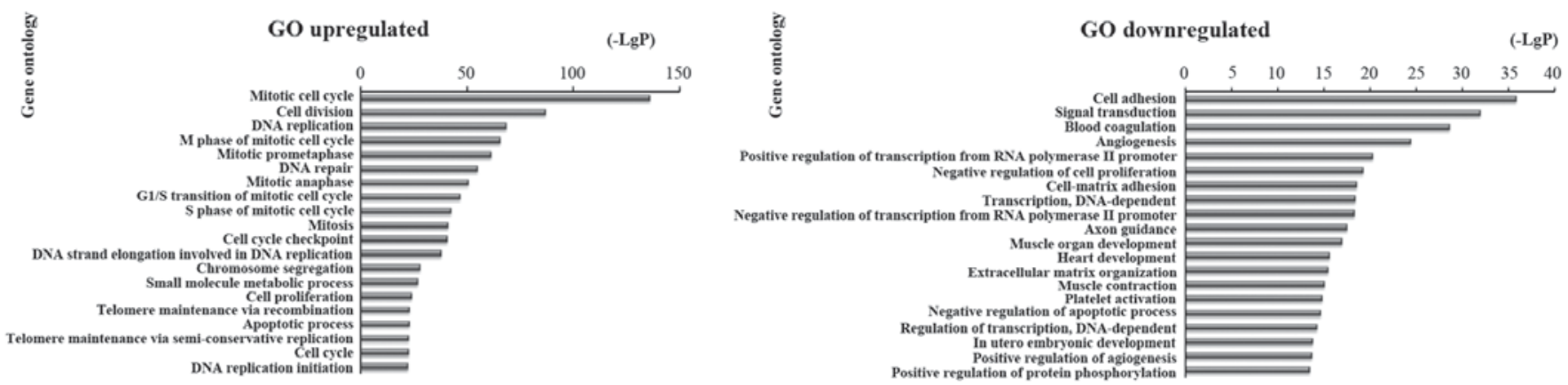

Figure 3. Top 20 enrichment of GO terms for aberrantly expressed intersection mRNAs. The bar plot indicated the enrichment scores of the significant top 20 enrichment GO terms. GO, Gene Ontology.

normal cervical tissues. In order to further narrow the scope of bioinformatics analysis and improve the accuracy, we chosed 51 lncRNAs that were common to all three groups (Fig. 2). There were 42 IncRNAs (13 upregulated; 29 downregulated; Table I) involved in the ceRNA network in these 51 lncRNAs.

Functional enrichment analysis. The function of differentially expressed mRNAs in CC was analyzed at the GO and KEGG pathway levels by DAVID Bioinformatics tool. There were 2,650 differentially expressed mRNAs between $\mathrm{CC}$ tumor tissues and adjacent normal cervical tissues in FIGO stage form the TCGA. Focused on these differentially expressed genes, there were 2,484 differentially expressed mRNAs between CC tumor tissues and adjacent normal cervical tissues in FIGO stage I; 2,392 differentially expressed mRNAs in FIGO stage II and 2,650 differentially expressed mRNAs in FIGO stage III-IV. We analyzed the enrichment of these 2,057 differentially expressed mRNAs in the GO database (Fig. 2), then analyzed the upregulated and downregulated mRNAs. We found that the highest enriched GO terms were mitotic cell cycle, cell division, DNA replication and apoptotic process in upregulated transcripts. and cell adhesion, signal transduction, transcription and DNA-dependent in downregulated transcripts (Fig. 3).

There were 87 pathways corresponded to upregulated transcripts by pathway analysis; the main enriched pathway was the Cell cycle. In the 109 pathways in the downregulated transcripts; the main enriched pathway was cGMP-PKG signaling pathway. We separately described the top 20 KEGG pathways, including downregulated and upregulated genes (Fig. 4). Among these pathways, the p53 signaling pathway, viral carcinogenesis, PI3K-Akt signaling pathway, Ras signaling pathway, MAPK signaling pathway, mTOR signaling pathway and Rap1 signaling pathway may be related to development and prognosis of cancer. In addition, other pathways such as cGMP-PKG signaling pathway, Cell cycle and leukocyte transendothelial migration were also associated with cancer pathways (Table II and Fig. 4).

The ceRNA network. In our study, we found 72 differentially expressed miRNAs with the fold-change $>2$ and $\mathrm{P}<0.05$. We picked out 58 intersection miRNAs from these 72 miRNAs by bioinformatics analysis of the FIGO stage (Fig. 2B). and determined if these interacting miRNAs had a target relationship with any of the $51 \mathrm{CC}$-specific lncRNAs. We predicted 56 miRNAs targeted 49 key lncRNAs by miRcode (http://www.mircode.org/) (23) (Table III) in the ceRNAs network. Then, mRNA targeted by miRNAs, we found 49 specific miRNAs associated with 97 mRNAs (Tables II and IV). Some mRNAs targeted cancer-associated genes, including BCL2, MAP3K3, AKT3, E2F3.

Based on our bioinformatics analysis, we investigate the relationship between IncRNAs and mRNAs potential 
Table II. KEGG pathways enriched by the coding genes involved in the competing endogenous RNA network.

A, Upregulated genes

KEGG pathways

Genes

Cancer related

Pathways in cancer, MicroRNAs in cancer, prostate cancer, p53 signaling pathway, PI3K-Akt signaling pathway, small cell lung cancer, HIF-1 signaling pathway, viral carcinogenesis

E2F3, TPM3, MYB, NUP188, CCNE1, CHEK1, EPHA1, NUP50, SRPK1, WWC1, E2F3, EXO1, NXT2, ACACA, CDC25A, GALNT3, SLC2A1, TCF7, XPO5, ELK4, PDE7A, PAK6, PIGA, BCL2L11, HK2

Non-cancer related

HTLV-I infection, cell cycle, RNA transport, renal cell carcinoma, axon guidance

B, Downregulated genes

KEGG pathways

Genes

Cancer related

Pathways in cancer, Rap1 signaling pathway, PI3K-Akt signaling pathway, Ras signaling pathway, MAPK signaling pathway, prostate cancer, ErbB signaling pathway, mTOR signaling pathway, endometrial cancer, small cell lung cancer

CALD1, DOCK4, FLT1, GAB1, GUCY1A3, KCNJ8, KCNMA1, NR4A3, PDGFRA, PPP1R12B, PTGER3, RPS6KA2, S1PR1, SLC2A4, ST6GALNAC3, ST6GALNAC6, ZFPM2, HGF, PRKCA, PTGER2, STAT5B, ZAK, AXIN2, BCL2, FGF2, INSR, MASP1, PRLR, RAB11FIP2, RECK, SGCD, ZYX, NRXN3, ZEB1, ZEB2, CTSK, ESAM, THRA, ACACB, GNAZ, SDC2, AKT3, SPG20, TBL1X, CALD1, ABCC9, MYLK, ST3GAL2, KDR, MEF2D, ACTC1, CACNB2, ERG, DUSP3, GNG7, MAP3K3, NCAM1, SOX17, ST3GAL3, ADCY5, FGFR1, FZD4, ITGA10, PRKG1, ATP2B4, HOXA11, MITF, ST8SIA1, ENTPD1, MAGI2, MEF2C, AMPH, NEGR1

Non-cancer related

cGMP-PKG signaling pathway, focal adhesion, transcriptional misregulation in cancer, insulin resistance, apoptosis, leukocyte transendothelial migration

KEGG, Kyoto Encyclopedia of Genes and Genomes.
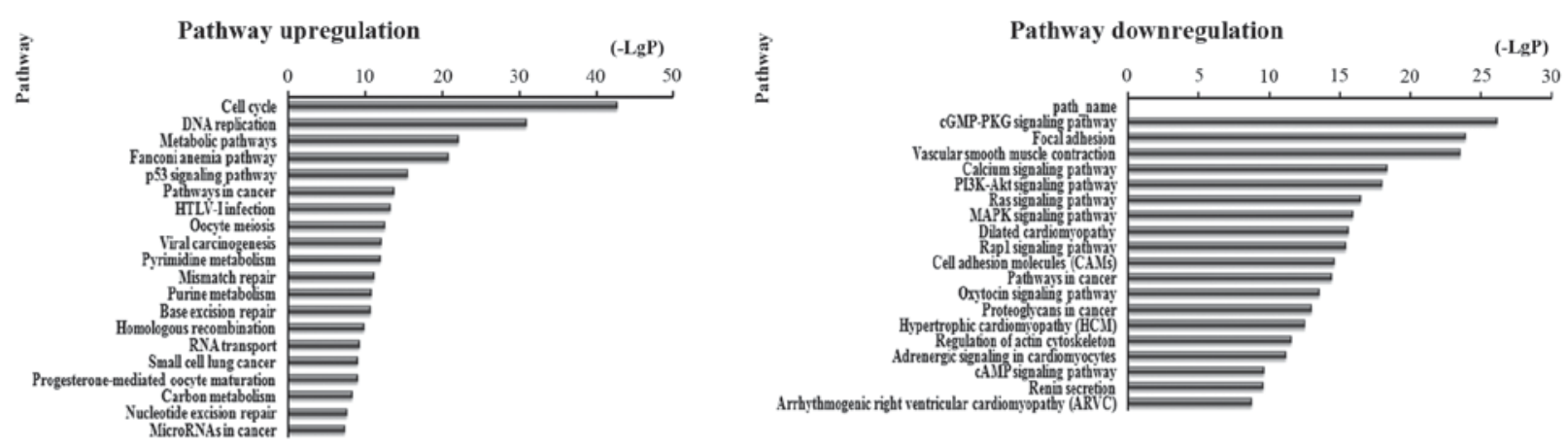

Figure 4. Top 20 enrichment of KEGG pathways for aberrantly expressed intersection mRNAs (the bar plot shows the enrichment scores of the significant top 20 enrichment KEGG pathways). KEGG, Kyoto Encyclopedia of Genes and Genomes.

linked by miRNAs that were identified in Tables II and III, and build the ceRNA (IncRNA-miRNA-mRNA) network. There were 72 differentially expressed miRNAs identified in $\mathrm{CC}$ tissues samples, among which were the 58 intersecting
miRNAs (Fig. 2B). We then used the MREs principle to find the relationships between these 58 miRNAs and $51 \mathrm{CC}$-specific IncRNAs, and detected the potential MREs by starBase. The results showed that there were 49 specific 
Table III. miRNAs targeting specific intersection key lncRNAs in CC.

Key lncRNAs

miRNAs

A2M-AS1

ACVR2B-AS1

AMZ2P1

ASMTL-AS 1

ATP1A1-AS1

CDKN2B-AS1

DDX12P

EMX2OS

EP400NL

EPB41L4A-AS1

FAM66C

FLJ10038

FTX

GEMIN8P4

GOLGA2P10

GOLGA2P5

ILF3-AS 1

INE2

LINC00312

LINC00341

LINC00467

LINC00663

LINC00950

LINC01140

LOC 146880

LOH12CR2

MBNL1-AS1

MEG3

MIR100HG

MIR4697HG

MIR9-3HG

MIR99AHG

MST1P2

NR2F1-AS1

OIP5-AS1

RASA4CP

SMIM10L2B

SNHG7

SYS1-DBNDD2

TMPO-AS 1

TPTEP1

ZNF876P
hsa-miR-183-5p, hsa-miR-93-5p

hsa-miR-106b-5p, hsa-miR-15b-5p, hsa-miR-93-5p

hsa-miR-183-5p

hsa-miR-30b-5p

hsa-miR-106b-5p, hsa-miR-183-5p

hsa-miR-140-5p, hsa-miR-195-5p

hsa-miR-10b-3p, hsa-miR-139-5p, hsa-miR-140-3p, hsa-miR-145-5p, hsa-miR-497-5p

hsa-miR-106b-5p, hsa-miR-141-5p, hsa-miR-16-5p, hsa-miR-183-5p, hsa-miR-205-5p,

hsa-miR-21-3p, hsa-miR-93-5p

hsa-miR-140-3p

hsa-miR-141-5p, hsa-miR-15b-5p, hsa-miR-16-5p

hsa-miR-15b-5p, hsa-miR-16-5p, hsa-miR-185-5p

hsa-miR-106b-5p, hsa-miR-183-5p, hsa-miR-200b-3p, hsa-miR-32-5p, hsa-miR-429

hsa-miR-185-5p

hsa-miR-143-3p

hsa-miR-10b-5p, hsa-miR-133a-3p, hsa-miR-140-3p, hsa-miR-195-5p, hsa-miR-320a, hsa-miR-497-5p hsa-miR-132-3p, hsa-miR-133a-3p, hsa-miR-139-5p, hsa-miR-328-3p

hsa-miR-106b-5p, hsa-miR-93-5p

hsa-miR-106b-5p, hsa-miR-93-5p

hsa-miR-15b-5p, hsa-miR-16-5p, hsa-miR-21-3p

hsa-miR-200a-3p, hsa-miR-205-5p, hsa-miR-425-5p

hsa-miR-132-3p, hsa-miR-133a-3p

hsa-miR-106b-5p, hsa-miR-141-3p, hsa-miR-15b-5p, hsa-miR-200a-3p, hsa-miR-93-5p

hsa-miR-141-3p, hsa-miR-141-5p, hsa-miR-200b-3p, hsa-miR-200c-3p, hsa-miR-224-5p,

hsa-miR-142-3p, hsa-miR-21-3p

hsa-miR-142-3p, hsa-miR-21-3p

hsa-miR-145-5p

hsa-miR-106b-5p, hsa-miR-93-5p

hsa-miR-106b-5p, hsa-miR-141-3p, hsa-miR-183-5p, hsa-miR-200a-3p

hsa-miR-32-5p, hsa-miR-93-5p

hsa-miR-106b-5p, hsa-miR-22-5p, hsa-miR-429, hsa-miR-93-5p

hsa-miR-183-5p

hsa-miR-141-5p, hsa-miR-205-5p, hsa-miR-22-5p

hsa-miR-10b-5p, hsa-miR-139-5p, hsa-miR-140-5p, hsa-miR-143-5p, hsa-miR-195-5p, hsa-miR-320a hsa-miR-106b-5p, hsa-miR-141-5p, hsa-miR-182-5p, hsa-miR-93-5p

hsa-miR-328-3p

hsa-miR-141-5p, hsa-miR-15b-5p, hsa-miR-185-5p, hsa-miR-22-5p, hsa-miR-425-5p

hsa-miR-143-5p

hsa-miR-182-5p

hsa-miR-15b-5p, hsa-miR-182-5p, hsa-miR-205-5p, hsa-miR-425-5p

hsa-miR-182-5p, hsa-miR-200a-5p

hsa-miR-16-5p

hsa-miR-143-3p

hsa-miR-141-3p, hsa-miR-142-3p, hsa-miR-16-5p

hsa-miR-106b-5p, hsa-miR-15b-3p, hsa-miR-93-5p

CC, cervical cancer; lncRNA, long non-coding RNA.

miRNAs and 42 specific lncRNAs with potential regulatory relationships. We then used Cytoscape 3.0 to build the ceRNA network based on data from Tables III and IV.
Fig. 5 shows the 42 lncRNAs, 49 miRNAs, and 72 mRNAs participating in the IncRNA-miRNA-mRNA interaction network of CC. 
Table IV. miRNAs targeting CC-specific mRNAs.

miRNAs

hsa-miR-106b-5p

hsa-miR-10b-3p hsa-miR-10b-5p hsa-miR-125a-5p

hsa-miR-125b-5p hsa-miR-126-5p hsa-miR-132-3p hsa-miR-133a-3p hsa-miR-139-5p hsa-miR-140-3p hsa-miR-140-5p hsa-miR-141-3p

hsa-miR-141-5p hsa-miR-142-3p hsa-miR-143-3p hsa-miR-143-5p hsa-miR-145-3p hsa-miR-145-5p hsa-miR-15b-3p hsa-miR-15b-5p

hsa-miR-16-5p

hsa-miR-182-5p hsa-miR-183-5p hsa-miR-185-5p hsa-miR-195-5p

hsa-miR-200a-3p

hsa-miR-200a-5p hsa-miR-200b-3p

hsa-miR-200c-3p

hsa-miR-205-5p

hsa-miR-21-3p

hsa-miR-218-5p

hsa-miR-22-5p

hsa-miR-224-5p

hsa-miR-24-1-5p

hsa-miR-28-3p

hsa-miR-28-5p

hsa-miR-30b-5p

hsa-miR-32-5p

hsa-miR-320a

hsa-miR-328-3p

hsa-miR-361-5p hsa-miR-362-5p
mRNAs

BCL2L11, CALD1, DOCK4, E2F2, E2F3, ELK4, ERBB3, FLT1, GAB1, GUCY1A3, KCNJ8, KCNMA1, KPNA2, NR4A3, PDGFRA, PPP1R12B, PTGER3, RPS6KA2, RUNX1, S1PR1, SLC2A

MAGI2, MEF2D, PRLR, RHOQ, XPO5

E2F3, NR4A3, SHANK3

BAK1, BCL2, CDKN2B, DUSP3, E2F2, EIF4EBP1, ENPP1, FGFR1, LIFR, MAP3K3, MASP1, NUP210, NUP50, PIP5K1C, PPAT, PPP1R12B, RHOQ, SCN4B, TDG

ACACB, BAK1, BCL2, CDKN2B, E2F2, LIFR, MAP3K3, NUP210, PPAT, PPP1R12B, TDG, TSTA3 PDGFRA

FGF7, MAP3K3, PDE7A, PPP2CB, PRICKLE2

AQP1, DAAM2, GABARAPL1, SGCD, TBL1X, TPM3

ANK2, DMD, FOXO1, GALNT3, MRVI1, SOCS2, TPM3

BCL2, GAB2, KCNMA1, MYB, NUP188, VAMP2

ACACA, DNM3, PDGFRA, SLC2A1

CDC25A, DUSP3, E2F3, ERG, GNG7, HGF, MAP3K3, NCAM1, NME1, PIGW, RUNX1, SOX17, ST3GAL3, ZEB1, ZEB2

HGF, HSP90AA1, NUP50, PRKCA, PTGER2, STAT5B, ZAK

PRLR

CACNA1C, HK2, LIFR, NCAM1

RHOQ, TCF7, ZAK

DUSP3, ITGA10, PDE7B

ELK4, FLI1, FLT1, FZD4, PARVA, PTGFR, ST6GALNAC3, TGFBR2

CGN, NEGR1

ACACA, ADCY5, AKT3, AXIN2, BCL2, CCNE1, CHEK1, E2F3, EPHA1, FGFR1, FOXO1, FZD4, INSR, ITGA10, KDR, MASP1, MYB, NUP50, PPP1R12B, PRKG1, RAB11FIP2, RECK, SGCD, SRPK1, WWC1, ZYX

AXIN2, BCL2, CCNE1, CHEK1, E2F3, EPHA1, FGF2, FOXO1, INSR, MASP1, MYB, NUP50, PPP1R12B, PRLR, RAB11FIP2, RECK, SGCD, UNG, WWC1, ZYX

BCL2, DSG2, MEF2D, MITF, NUP50, PRLR, PTGER3, RECK, ST6GALNAC3, ST8SIA1, UCK2 EZR, FOXO1, NRXN3, TPM3, ZEB1, ZEB2, ZFPM2

CTSK, ESAM, PAK6, THRA

BCL2, CCNE1, CHEK1, EPHA1, FGF2, FGF7, FOXO1, FZD4, GABARAPL1, MASP1, MYLK, NUP50, PPP1R12B, PRLR, RAB11FIP2, SRPK1, WWC1, ZYX

B3GNT5, CDC25A, DUSP3, E2F3, ERG, GAB1, MAP3K3, NME1, RUNX1 SOX17, ST3GAL3, ZEB1, ZEB2

FGFR1, POLA1

ABCC9, DOCK4, E2F3, ELK4, GAB1, MYLK, PPP1R12B, RAB11FIP2, RUNX1, ST3GAL2, TP73, ZEB1, ZFPM2

DOCK4, ELK4, KDR, MEF2D, MYLK, PMAIP1, PPP1R12B, PRKCA, PTGER2, RAB11FIP2, RECK, RUNX1, ST3GAL2, TP73, ZEB1, ZEB2, ZFPM2

ACACB, DHCR24, E2F1, ERBB3, TGFA

GNAZ, NRXN3, RPS6KA2, SDC2, UCK2

APH1B, BRCA1, ELK4, GAB2, MTMR1, PRLR

ELK4, ENTPD1, MAGI2, MEF2C, RAD54B, SDC1

ATP2B4, HOXA11, KCNMA1, LPAR5, NR4A3

CALD1, DNM3, E2F3, TPM3

$\mathrm{LMO} 7$

ITPKB, MASP1, MPL, PARVA

BCL2L11, CACNA1C, DMD, GALNT3, MEF2D, PRLR

ACTC1, AURKA, BCL2L11, E2F3, ELK4, SDC2, SLX4, ZEB2

AKT3, CACNA1C, E2F3, EXO1, FLNC, GNAZ, GUCY1A3, NXT2, PRKG1, TPM3

PAK6, PIGA, RASGRP2, SLC2A1, ST3GAL3, ZAK

ERG, GTF2E1, PIGA, PRICKLE2, ST8SIA1

AKT3, ATP2B4, KCNMA1, MRVI1, NRXN3 
Table IV. Continued.

miRNAs

mRNAs

hsa-miR-374b-5p

hsa-miR-381-3p

hsa-miR-425-5p

hsa-miR-429

hsa-miR-497-5p

hsa-miR-93-5p
FBXO32

CACNA1C, ELK4, FOXO1, GABARAPL1, ZFPM2

$\mathrm{AMPH}$

CACNB2, DOCK4, E2F3, ELK4, ERG, GAB1, GTF2E1, GUCY1A3, MYB, RAB11FIP2, RUNX1, ST3GAL2, TP73, ZEB1, ZFPM2

ACACA, ADCY5, AKT3, BCL2, CDC25A, CNTNAP1, E2F3, EPHA1, FGF2, FOXO1, FZD4, INSR, ITGA10, KDR, MASP1, MYLK, NUP50, PTPRM, RAB11FIP2, RECK, SGCD, SRPK1, WWC1, ZAK, ZYX

AKT3, BCL2L11, E2F1, E2F2, ELK4, ERBB3, FLT1, GAB1, GUCY1A3, KCNJ8, KCNMA1, KIF23, KPNA2, NR4A3, PGP, PPP1R12B, PTGER3, RBL1, RPS6KA2, RUNX1, SGCD, SLC2A4, SPG20, ST6GALNAC3, ST6GALNAC6, TBL1X, THRA

CC, cervical cancer.

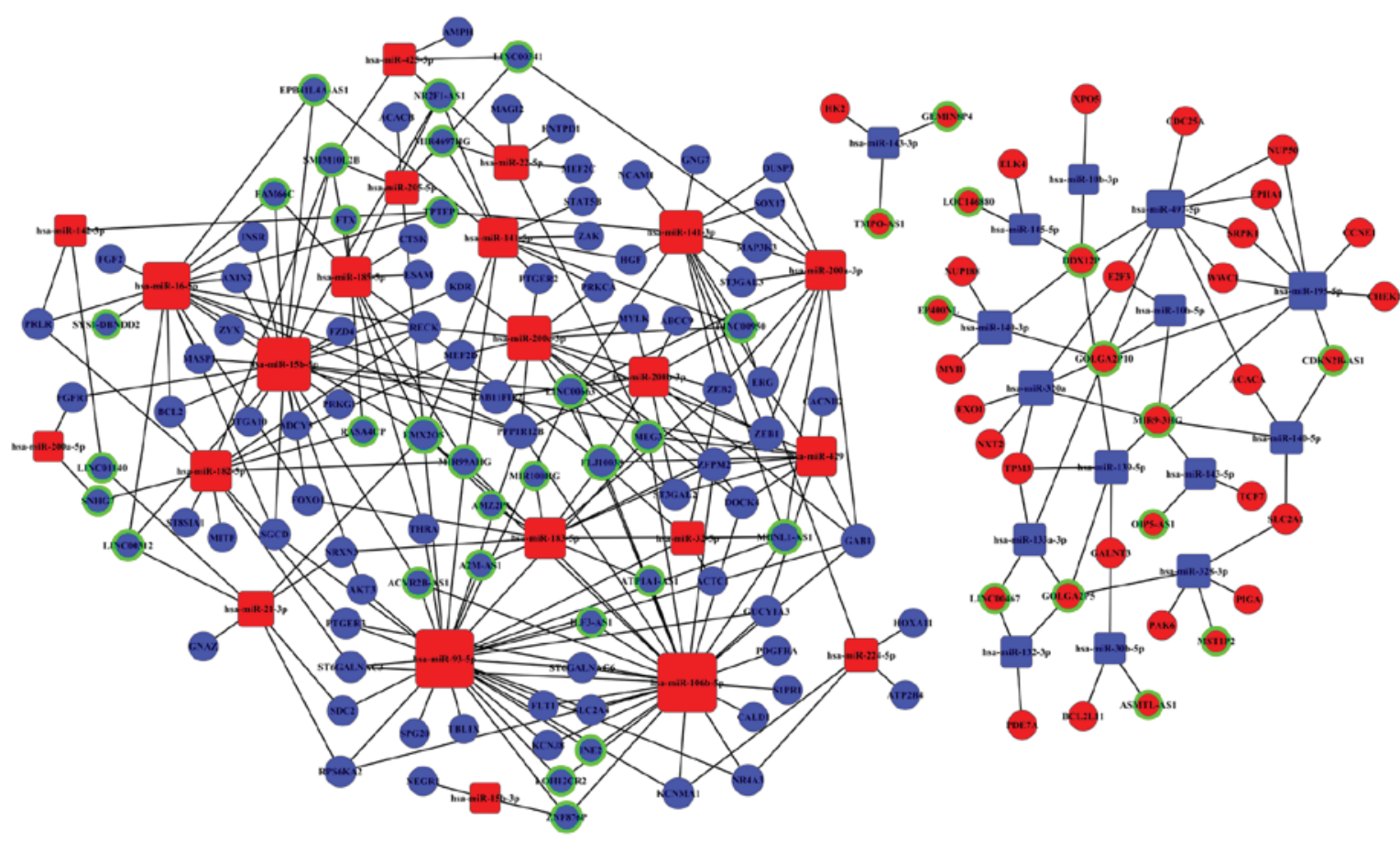

Figure 5. The lncRNA-miRNA-mRNA ceRNA network. Downregulated miRNAs (blue squares); upregulated miRNA (red squares); downregulated mRNAs (blue squares); upregulated mRNAs (red circles), downregulated lncRNAs (blue circles surrounded by green rings); upregulated lncRNAs (red circles surrounded by green rings). lncRNA, long non-coding RNA.

Correlation analysis between CC specific IncRNAs expression with clinical features. Using available clinical features from TCGA, such as race, tumor grade, TNM stage, clinical stage, HPV infection, and transfer, we further analyzed the 42 key lncRNAs from the ceRNA network. The expression levels of the 19 key lncRNAs were obviously different in patients with different clinical features $(\mathrm{P}<0.05$; Table V). For example two lncRNAs (MST1P2 and FTX) were differently expressed in CC patients of different race, five lncRNAs (LOH12CR2, GOLGA2P10, A2M-AS1, ATP1A1-AS1 and ACVR2B-AS1) were differently expressed at different pathological stage, ten IncRNAs (FAM66C, GOLGA2P5, ACVR2B-AS1, ZNF876P, MIR9-3HG, EMX2OS,
LINC00341, FLJ10038, ILF3-AS1 and AMZ2P1) were expressed differently depending on the tumor TNM stage, four lncRNAs (GOLGA2P5, ACVR2B-AS1, ZNF876P and MIR9-3HG) were differently expressed at different clinical stage, four lncRNAs (ILF3-AS1, GOLGA2P5, MIR9-3HG and FAM66C) were aberrantly expressed depending on the patient outcome assessment and four lncRNAs (SYS1-DBNDD2, MIR9-3HG, DDX12P, LINC00312) were differently expressed in high and low risk types of HPV infection (Table V).

$R T$-qPCR verification and ROC. In order to prove the reliability of the above bioinformatics analysis results from 
Table V. The correlations between CC specific lncRNAs from ceRNA network and clinical features.

\begin{tabular}{lll}
\hline Comparisons & \multicolumn{1}{c}{ Downregulated } & Upregulated \\
\hline $\begin{array}{l}\text { Race (Caucasian vs. Asian) } \\
\text { Outcome (dead vs. alive) }\end{array}$ & ILF3-AS1, FAM66C & MST1P2, FTX \\
Transfer (N1 vs. N0) & FAM66C, ZNF876P, ACVR2B-AS1 & GOLGA2P5, MIR9-3HG, \\
Classification (stage 34 vs. stage 12) & LINC00341, EMX2OS, FLJ10038 & ILF3-AS1, AMZ2P1, GOLGA2P5 \\
Tumor pathological stage (T34 vs. T12) & LINC00341, EMX2OS, FLJ10038 & GOLGA2P10 \\
Tomor grade (g12 vs. g34) & LOH12CR2, A2M-AS1, ATP1A1-AS1, & \\
HPV infection (high-risk vs. low-risk) & ACVR2B-AS1 & MIR9-3HG, DDX12P \\
\hline
\end{tabular}

CC, cervical cancer; lncRNA, long non-coding RNA; ceRNA, competing endogenous RNA

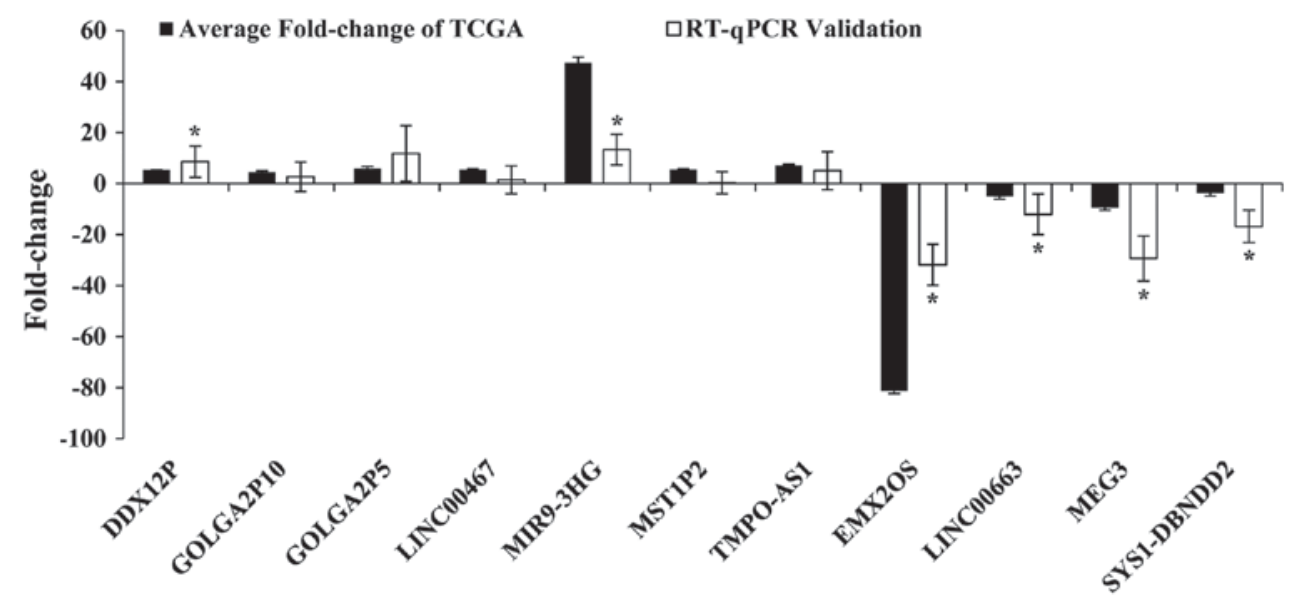

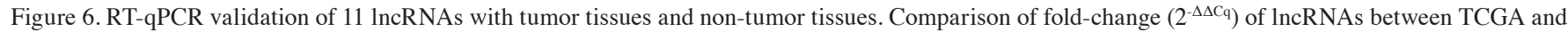
RT-qPCR results. IncRNA, long non-coding RNA; RT-qPCR, reverse transcription-quantitative polymerase chain reaction; TCGA, The Cancer Genome Atlas.

TCGA, we random selected 11 key lncRNAs (DDX12P, GOLGA2P5, GOLGA2P10, LINC00467, MIR9-3HG, MST1P2, TMPO-AS1, EMX2OS, LINC00663, MEG3, SYS1-DBNDD2) and verified their actual expression levels in 31 pairs of newly diagnosed clinical samples. The results showed that seven lncRNAs were upregulation and four IncRNAs were downregulated in CC tumor tissues compared to adjacent normal cervical tissues. The validation results for these 11 key lncRNAs were with the above TCGA bioinformatics results. This showed that our bioinformatics analysis was accurate and reliable (Fig. 6 and Table VI).

We assessed the diagnostic value of specific lncRNAs and found that three out of six lncRNAs examined displayed good diagnostic values (Fig. 7). ROC curve analysis revealed AUC values of $0.773,0.723$ and 0.724 for EMX20S, MEG3 and SYS1-DBNDD2, respectively $(\mathrm{P}<0.05$; Fig. 7A), which suggested that these lncRNAs may be good candidates for diagnostic biomarkers in CC because their AUC values exceeded 0.7. ROC analysis also showed an AUC value of 0.689 for MIR9-3HG ( $\mathrm{P}<0.05$; Fig. 7A), while results for DDX12P and LINC00663 were not statistically significant (P>0.05; Fig. 7C). The AUC of these four lncRNAs combined was 0.841 , which was higher than that of the single lncRNA $(\mathrm{P}<0.05$; Fig. 7B).

\section{Discussion}

Despite improvements in treatment, early prevention and diagnosis remains the most effective way to reduce morbidity and mortality of CC (24). With the extensive use of ThinPrep cytologic test (TCT) and HPV DNA screening techniques, the incidence and mortality rates of $\mathrm{CC}$ have declined over the past three decades, but the 5-year survival percentage of patients has still remained below $40 \%$ (4), and $85 \%$ deaths have occured in developing countries such as China (25). Therefore, the identification and validation of biomarkers for early diagnosis and prognosis of CC is an important goal. Many studies have reported lncRNAs related to the biological regulatory functions in many cancers (26). Abnormal expression of lncRNAs has also been widely detected in a variety of diseases $(23,27)$. Dysregulated lncRNAs have now emerged as key players in the development of cancer. However, the expression profiles of IncRNA in CC have been described in only a few studies involving small sample size (28). Furthermore, very few studies have examined the interaction between IncRNA, mRNA and miRNA in CC. Results from the few studies performed have showed that lncRNAs play an important function in ceRNA network, but their relationships to specific ceRNA networks are still unclear (29,30). Recently, a new ceRNA hypothesis was 
Table VI. Relative expression of lncRNAs in 31 pairs of cervical cancer tumor and non-tumor tissue.

\begin{tabular}{|c|c|c|c|c|c|c|c|}
\hline Gene symbol & Type & Group & $\begin{array}{l}\text { Mean } \pm \mathrm{SD} \\
\text { of } \Delta \mathrm{Cq}\end{array}$ & $\begin{array}{c}\Delta \Delta \mathrm{Cq}^{\mathrm{a}} \\
(\mathrm{mean} \pm \mathrm{SD})\end{array}$ & $2^{-\Delta \Delta \mathrm{Cq}}$ & P-value ${ }^{b}$ & t-value \\
\hline GOLGA2P10 & LncRNA & $\begin{array}{l}\text { Tumor tissues } \\
\text { Adjacent non-tumor } \\
\text { tissues }\end{array}$ & $\begin{array}{l}8.033 \pm 2.855 \\
7.849 \pm 2.211\end{array}$ & $0.184 \pm 2.521$ & 2.651 & 0.697 & 0.393 \\
\hline MIR9-3HG & LncRNA & $\begin{array}{l}\text { Tumor tissues } \\
\text { Adjacent non-tumor } \\
\text { tissues }\end{array}$ & $\begin{array}{l}11.173 \pm 2.732 \\
13.143 \pm 3.265\end{array}$ & $-2.008 \pm 2.602$ & 13.293 & $0.001^{\mathrm{b}}$ & 3.917 \\
\hline DDX12P & LncRNA & $\begin{array}{l}\text { Tumor tissues } \\
\text { Adjacent non-tumor } \\
\text { tissues }\end{array}$ & $\begin{array}{l}10.690 \pm 2.234 \\
11.853 \pm 2.488\end{array}$ & $-1.162 \pm 2.620$ & 8.523 & $0.026^{\mathrm{b}}$ & 2.347 \\
\hline GOLGA2P5 & LncRNA & $\begin{array}{l}\text { Tumor tissues } \\
\text { Adjacent non-tumor } \\
\text { tissues }\end{array}$ & $\begin{array}{l}10.164 \pm 2.348 \\
11.161 \pm 2.338\end{array}$ & $-0.789 \pm 3.451$ & 11.794 & 0.160 & 1.451 \\
\hline LINC00467 & LncRNA & $\begin{array}{l}\text { Tumor tissues } \\
\text { Adjacent non-tumor } \\
\text { tissues }\end{array}$ & $\begin{array}{l}11.645 \pm 2.066 \\
11.546 \pm 2.429\end{array}$ & $0.098 \pm 2.448$ & 1.461 & 0.839 & 0.205 \\
\hline MST1P2 & LncRNA & $\begin{array}{l}\text { Tumor tissues } \\
\text { Adjacent non-tumor } \\
\text { tissues }\end{array}$ & $\begin{array}{l}11.717 \pm 3.025 \\
11.075 \pm 2.972\end{array}$ & $0.646 \pm 2.109$ & 0.287 & 0.139 & 1.531 \\
\hline TMPO-AS1 & LncRNA & $\begin{array}{l}\text { Tumor tissues } \\
\text { Adjacent non-tumor } \\
\text { tissues }\end{array}$ & $\begin{array}{l}10.215 \pm 2.397 \\
10.402 \pm 2.619\end{array}$ & $-0.187 \pm 2.897$ & 5.056 & 0.749 & 0.232 \\
\hline EMX2OS & LncRNA & $\begin{array}{l}\text { Tumor tissues } \\
\text { Adjacent non-tumor } \\
\text { tissues }\end{array}$ & $\begin{array}{l}16.678 \pm 3.390 \\
13.525 \pm 3.836\end{array}$ & $3.153 \pm 3.011$ & -31.829 & $0.000^{\mathrm{b}}$ & 5.021 \\
\hline MEG3 & LncRNA & $\begin{array}{l}\text { Tumor tissues } \\
\text { Adjacent non-tumor } \\
\text { tissues }\end{array}$ & $\begin{array}{r}10.082 \pm 2.958 \\
8.035 \pm 2.308\end{array}$ & $2.047 \pm 3.143$ & -29.352 & $0.001^{\mathrm{b}}$ & 3.566 \\
\hline LINC00663 & LncRNA & $\begin{array}{l}\text { Tumor tissues } \\
\text { Adjacent non-tumor } \\
\text { tissues }\end{array}$ & $\begin{array}{l}19.529 \pm 2.851 \\
18.024 \pm 3.357\end{array}$ & $1.506 \pm 2.993$ & -12.051 & $0.015^{\mathrm{b}}$ & 2.614 \\
\hline SYS1-DBNDD2 & LncRNA & $\begin{array}{l}\text { Tumor tissues } \\
\text { Adjacent non-tumor } \\
\text { tissues }\end{array}$ & $\begin{array}{l}4.566 \pm 1.748 \\
3.029 \pm 2.175\end{array}$ & $1.537 \pm 2.676$ & -16.796 & $0.005^{\mathrm{b}}$ & 3.039 \\
\hline
\end{tabular}

${ }^{\mathrm{a}} \Delta \mathrm{Cq}=\mathrm{Cq}_{\text {target gene }}-\mathrm{Cq}_{\mathrm{GAPDH}} ; \Delta \Delta \mathrm{Cq}=\Delta \mathrm{Cq}_{\text {tumor tissues }}-\Delta \mathrm{Cq}_{\text {Adjacent non-tumor tissues }} .{ }^{\mathrm{b}} \mathrm{P}<0.05$.

proposed in which lncRNAs play a regulatory role through the competitive binding of miRNAs $(31,32)$. Based on this mechanism, Li et al constructed a ceRNA network related to oral squamous cell carcinoma (19). With further study of ceRNA network, many researchers have showen that miRNAs regulated gens and interact with lncRNAs in the ceRNA network (33).

In our study, we first screened lncRNAs, miRNAs and mRNAs. The three types of non-coding RNA were related to FIGO clinical stage in CC from the TCGA database. As far as we know, this is the first time that IncRNA-miRNA-mRNA ceRNA networks have been established in CC. Based on clinical information and RNA sequencing profiles, we found that specific key lncRNAs from ceRNA network were altered in different $\mathrm{CC}$ clinical manifestations by. We further verified the expression level of 11 key lncRNAs in clinical samples by RT-qPCR.
We investigated aberrantly expressed mRNAs in CC intersection with RNAs from the three groups of RNA sequence data. The results of GO and pathway analysis also revealed potential regulatory relationship of mRNA related lncRNAs. The abnormal signaling pathways may play important roles in the development and progression of CC The GO results showed significant differences in cellular functions and transcription process. The KEGG pathway analysis showed that PI3K-Akt signaling pathway $(34,35)$, p53 signaling pathway (36), MAPK signaling pathway, and viral carcinogenesis were particularly important cancer-related pathways (37).

An increasing number of studies have also showed that lncRNAs may bind to other transcription factors and are involve in regulating the ceRNA network $(14,38,39)$. For example, the lncRNA MEG3 is an important gene for the progression of many types of cancer including CC (40). MEG3 
A

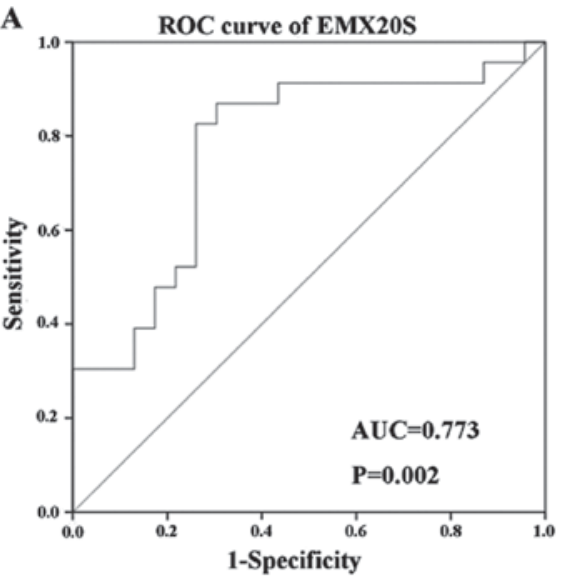

ROC curve of MIR9-3HG

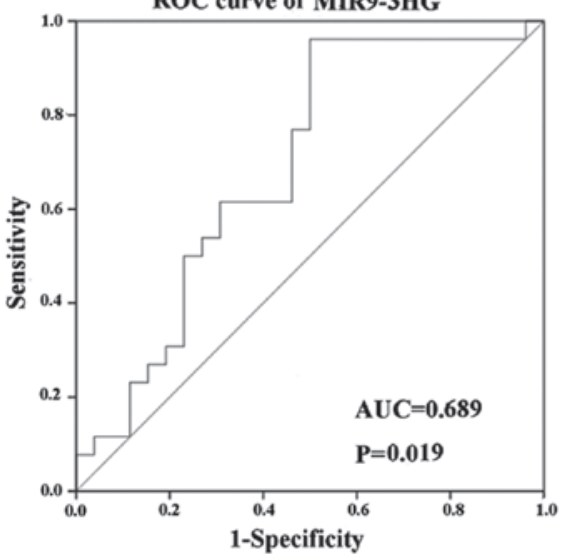

B

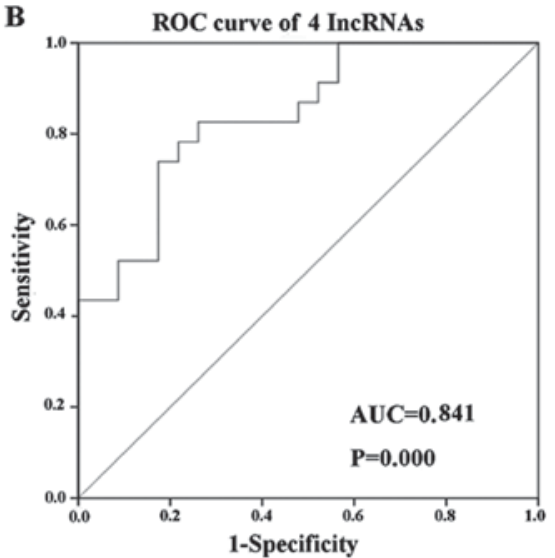

C

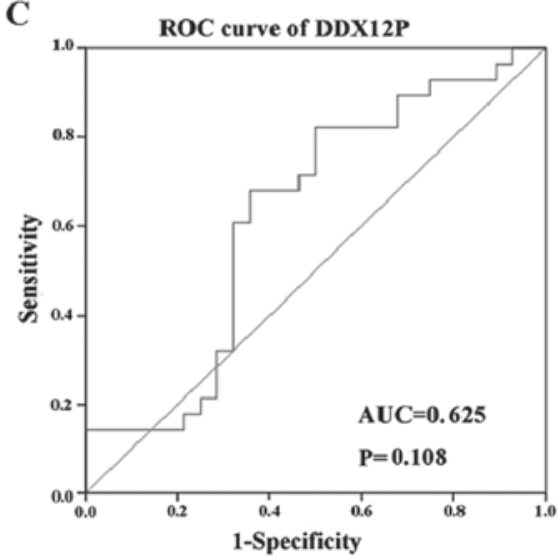

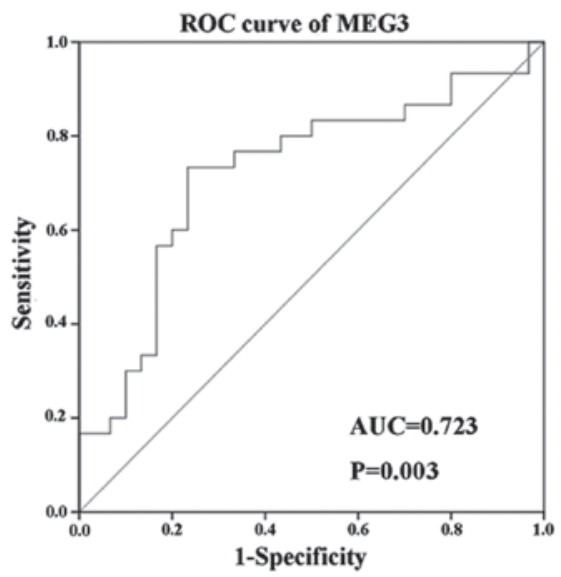

ROC curve of SYS1-DBNDD2

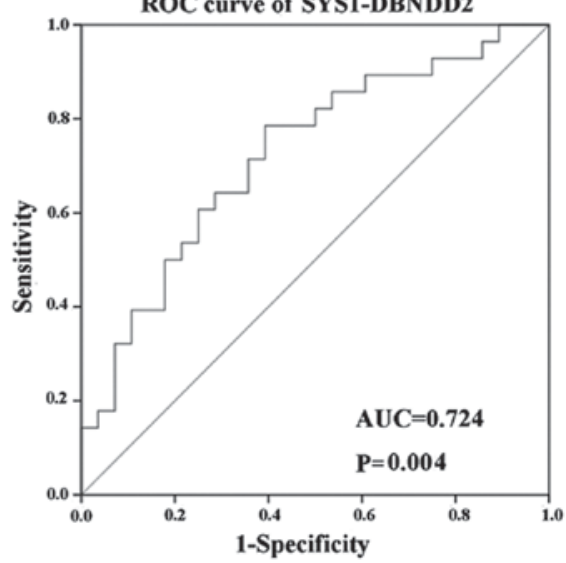


over-expression imposes another level of post-transcriptional regulation, whereas MEG3 over expression increase the expression of the miR-664 target gene, ADH4, through competitive sponging of miR-664. Therefore, the potential regulatiory function of lncRNA-miRNA-mRNA interactions may also act during $\mathrm{CC}$ development. Based on the above analysis, we built an IncRNA-miRNA-mRNA ceRNA network in CC through bioinformatics analysis. We found that particular lncRNAs may be associated with cancer. The lncRNAs such as MEG3, LINC00341 and LINC00663 (41-43) may therefore acted as potential molecular biomarker in other cancers, and may also be involved in the initiation and progression of cancer. Based on our research analysis, specific lncRNA was found to be indirectly related to mRNAs signaling pathways in ceRNA network of CC. The analysis results showed at leaet 10 pathways connected to cancer. Therefore, it is believed that these key lncRNAs may played an important regulatory role during $\mathrm{CC}$ formation.

We analyzed the association of 42 key lncRNAs from the ceRNA network. The 19 key lncRNAs were related to clinical features. According to recent studies, these included the IncRNAs LINC00341 (42), FTX (44), LOH12CR2 (45) and LINC00312 (46), which have been reported to be associated with prognosis in several cancers, while the function of other lncRNAs have not yet been reported. These lncRNAs, which were associated with clinical features, may have important research values in the development and prognosis of CC. We also uesed RT-qPCR to verify the expressions level of 11 key lncRNAs from the 31 pairs of newly obtained clinical samples. The result of RT-qPCR were consistent with the result of TCGA bioinformatics analysis, showing that it was basically reliable. The specificity and sensitivity of lncRNAs as a test indicator were then determined by ROC. Three lncRNAs (EMX20S, MEG3, SYS1-DBNDD2) had significant single diagnostic values, but more important, the AUC of the combined four lncRNAs (EMX20S, MEG3, SYS1-DBNDD2, MIR9-3HG) was 0.841 $(\mathrm{P}<0.05)$, which was greater than that any single lncRNA, suggested that the combined diagnosis could improve the diagnostic efficacy of CC.

In conclusion, we screened for key lncRNAs which related to $\mathrm{CC}$ from the large number of candidate lncrRNAs in the TCGA database by bioinformatics analysis and found differentially expressed lncRNAs associated with different clinical features. Importantly, we have constructed a ceRNA network which encompassed the IncRNA-miRNA-mRNA interactions in $\mathrm{CC}$, and investigated the CC related key lncRNAs for their potential regulatory role. We also validated key lncRNAs expression levels by RT-qPCR and thus demonstrated the reliability and validity our bioinformatics analysis. Furthermore, we explored the diagnostic value of some these key lncRNAs. Our results suggested that these key lncRNAs may be new candidate biomarkers for the clinical diagnosis, classification and prognosis of CC. Due to sample size limitations of TCGA database. Preliminary analysis and screening was only a reference and exploration, our research focused on the follow-up study for the enlarged sample size of Chinese population. Future research studies will require molecular investigations and more clinical samples to verify the function and mechanism of these lncRNAs.

\section{Acknowledgements}

The authors would like to thank Mr. Donglin Cheng for his technical support.

\section{Funding}

This study was supported by the National Natural Science Foundation of China (grant nos. 81673132, 81673130 and 81472939), the 333 Project of Jiangsu Province, the Fundamental Research Funds for the Central Universities and the Innovative Research Project for Postgraduates in Colleges of Jiangsu Province.

\section{Availability of data and materials}

The datasets used and/or analyzed during the current study are available from the corresponding author on reasonable request.

\section{Author's contributions}

WJW and GYL conceived and designed the study. WJW and CYL performed the experiments. WJW, CYL, JS, SY, SYX and $\mathrm{MZ}$ analyzed and interpreted the results. YS performed the cervical cancer patients' tissue sample collection and quality control. LHY and YPP assisted with study design and provided advice throughout. WJW performed analysis and quality control and was a major contributor in writing the manuscript. All authors read and approved the final manuscript.

\section{Ethics approval and consent to participate}

The present study was approved by the Ethics Committee of the Zhongda Hospital of Southeast University (Nanjing, China). All patients provided written informed consent to participate in the present study.

\section{Consent for publication}

Not applicable.

\section{Competing interests}

All authors declare that they have no competing interests.

\section{References}

1. Sankaranarayanan R and Ferlay J: Worldwide burden of gynaecological cancer: The size of the problem. Best Pract Res Clin Obstet Gynaecol 20: 207-225, 2006.

2. Kim HJ, Lee DW, Yim GW, Nam EJ, Kim S, Kim SW and Kim YT: Long non-coding RNA HOTAIR is associated with human cervical cancer progression. Int J Oncol 46: 521-530, 2015.

3. Bray F, Ren JS, Masuyer E and Ferlay J: Global estimates of cancer prevalence for 27 sites in the adult population in 2008. Int J Cancer 132: 1133-1145, 2013.

4. O'Mara TA, Zhao M and Spurdle AB: Meta-analysis of gene expression studies in endometrial cancer identifies gene expression profiles associated with aggressive disease and patient outcome. Sci Rep 6: 36677, 2016.

5. Dey BK, Mueller AC and Dutta A: Long non-coding RNAs as emerging regulators of differentiation, development and disease. Transcription 5: e944014, 2014. 
6. Mercer TR and Mattick JS: Structure and function of long noncoding RNAs in epigenetic regulation. Nat Struct Mol Biol 20: 300-307, 2013.

7. Peng L, Yuan XQ, Liu ZY, Li WL, Zhang CY, Zhang YQ, Pan X, Chen J, Li YH and Li GC: High lncRNA H19 expression as prognostic indicator: data mining in female cancers and polling analysis in non-female cancers. Oncotarget 8: 1655-1667, 2017.

8. Li CY, Liang GY, Yao WZ, Sui J, Shen X, Zhang YQ, Peng H, Hong WW, Ye YC, Zhang ZY, et al: Identification and functional characterization of microRNAs reveal a potential role in gastric cancer progression. Clin Transl Oncol 19: 162-172, 2017.

9. Liu J, Liu L, Wan JX and Song Y: Long noncoding RNA SNHG20 promotes gastric cancer progression by inhibiting p21 expression and regulating the GSK- $3 \beta / \beta$-catenin signaling pathway. Oncotarget 8: 80700-80708, 2017.

10. Peng Z, Wang J, Shan B, Yuan F, Li B, Dong Y, Peng W, Shi W, Cheng Y, Gao Y, et al: Genome-wide analyses of long noncoding RNA expression profiles in lung adenocarcinoma. Sci Rep 7: $15331,2017$.

11. Fu Y, Biglia N, Wang Z, Shen Y, Risch HA, Lu L, Canuto EM, Jia W, Katsaros D and Yu H: Long non-coding RNAs, ASAP1-IT1, FAM215A and LINC00472, in epithelial ovarian cancer. Gynecol Oncol 143: 642-649, 2016.

12. Sun R, Qin C, Jiang B, Fang S, Pan X, Peng L, Liu Z, Li W, Li Y and Li G: Down-regulation of MALAT1 inhibits cervical cancer cell invasion and metastasis by inhibition of epithelial-mesenchymal transition. Mol Biosyst 12: 952-962, 2016.

13. Li Z, Chen Y, Hu S, Zhang J, Wu J, Ren W, Shao N and Ying X: Integrative analysis of protein-coding and non-coding RNAs identifies clinically relevant subtypes of clear cell renal cell carcinoma. Oncotarget 7: 82671-82685, 2016.

14. Song X, Cao G, Jing L, Lin S, Wang X, Zhang J, Wang M, Liu W and Lv C: Analysing the relationship between lncRNA and protein-coding gene and the role of lncRNA as ceRNA in pulmonary fibrosis. J Cell Mol Med 18: 991-1003, 2014.

15. Salmena L, Poliseno L, Tay Y, Kats L and Pandolfi PP: A ceRNA hypothesis: The Rosetta Stone of a hidden RNA language? Cell 146: 353-358, 2011

16. Martin CM, Astbury K, Mcevoy L, O'Toole S, Sheils O and O'Leary JJ: Gene expression profiling in cervical cancer: Identification of novel markers for disease diagnosis and therapy. Methods Mol Biol 511: 333-359, 2009.

17. Goldfeder RL, Wall DP, Khoury MJ, Ioannidis JPA and Ashley EA: Human genome sequencing at the population scale: A primer on high-throughput DNA sequencing and analysis. Am J Epidemiol 186: 1000-1009, 2017.

18. Shukla HD: Comprehensive analysis of cancer-proteogenome to identify biomarkers for the early diagnosis and prognosis of cancer. Proteomes 5: E28, 2017.

19. Li S, Chen X, Liu X, Yu Y, Pan H, Haak R, Schmidt J, Ziebolz D and Schmalz G: Complex integrated analysis of IncRNAs-miRNAs-mRNAs in oral squamous cell carcinoma. Oral Oncol 73: 1-9, 2017

20. Guo LL, Song CH, Wang P, Dai LP, Zhang JY and Wang KJ: Competing endogenous RNA networks and gastric cancer. World J Gastroenterol 21: 11680-11687, 2015.

21. Lu H, Shi B, Wu G, Zhang Y, Zhu X, Zhang Z, Liu C, Zhao Y, Wu T, Wang $\mathrm{J}$ and Chen R: Integrated analysis of multiple data sources reveals modular structure of biological networks. Biochem Biophys Res Commun 345: 302-309, 2006.

22. Zheng Y, Wei L, Guo L and Yang X: The expression level of miR-203 in patients with gastric cancer and its clinical significance. Pathol Res Pract 13: 1515-1518, 2017.

23. Miao Y, Xu SY, Chen LS, Liang GY, Pu YPG and Yin LH: Trends of long noncoding RNA research from 2007 to 2016: A bibliometric analysis. Oncotarget 8: 83114-83127, 2017.

24. Siegel R, Naishadham D and Jemal A: Cancer statistics, 2012. CA Cancer J Clin 62: 10-29, 2012

25. World Health Organization (WHO): New WHO guide to prevent and control cervical cancer. http://www.who.int/mediacentre/ news/releases/2014/preventing-cervical-cancer/en/. Accessed December 3, 2014

26. Moran VA, Perera RJ and Khalil AM: Emerging functional and mechanistic paradigms of mammalian long non-coding RNAs. Nucleic Acids Res 40: 6391-6400, 2012.
27. Hu X, Sood AK, Dang CV and Zhang L: The role of long noncoding RNAs in cancer: The dark matter matters. Curr Opin Genet Dev 48: 8-15, 2017.

28. Yang L, Yi K, Wang H, Zhao Y and Xi M: Comprehensive analysis of lncRNAs microarray profile and mRNA-lncRNA co-expression in oncogenic HPV-positive cervical cancer cell lines. Oncotarget 7: 49917-49929, 2016.

29. Wei G: Bioinformatics analysis of microRNA comprehensive regulatory network in congenital microtia. Int J Pediatr Otorhinolaryngol 79: 1727-1731, 2015

30. Qu J, Li M, Zhong $\mathrm{W}$ and $\mathrm{Hu} \mathrm{C}$ : Competing endogenous RNA in cancer: A new pattern of gene expression regulation. Int J Clin Exp Med 8: 17110-17116, 2015

31. Tay Y, Rinn J and Pandolfi PP: The multilayered complexity of ceRNA crosstalk and competition. Nature 505: 344-352, 2014.

32. Thomson DW and Dinger ME: Endogenous microRNA sponges: Evidence and controversy. Nat Rev Genet 17: 272-283, 2016.

33. Karreth FA and Pandolfi PP: ceRNA cross-talk in cancer: When ce-bling rivalries go awry. Cancer Discov 3: 1113-11121, 2013.

34. Zhang L, Wu J, Ling MT, Zhao L and Zhao KN: The role of the PI3K/Akt/mTOR signalling pathway in human cancers induced by infection with human papillomaviruses. Mol Cancer 14: 1, 2015.

35. Zhang D, Sun G, Zhang H, Tian J and Li Y: Long non-coding RNA ANRIL indicates a poor prognosis of cervical cancer and promotes carcinogenesis via PI3K/Akt pathways. Biomed Pharmacother 85: 511-516, 2017.

36. Rong Z, Lu H, Lyu YY, Yang XM, Zhu LY, Yang GD, Jiang PC, Re Y, Song WW, Wang JH, et al: E6/E7-P53-POU2F1-CTHRC1 axis promotes cervical cancer metastasis and activates Wnt/PCP pathway. Sci Rep 7: 44744, 2017.

37. Ling W, Zhigang H, Tian H, Bin Z, Xiaolin X and Hongxiu Z: HPV 16 infection up-regulates Piwil2, which affects cell proliferation and invasion in cervical cancer by regulating MMP-9 via the MAPK pathway. Eur J Gynaecol Oncol 36: 647-654, 2015.

38. Wu Q, Guo L, Jiang F, Li L, Li Z and Chen F: Analysis of the miRNA-mRNA-lncRNA networks in ER+ and ER- breast cancer cell lines. J Cell Mol Med 19: 2874-2887, 2015.

39. Li CY, Liang GY, Yao WZ, Sui J, Shen X, Zhang YQ, Peng H, Hong WW, Ye YC, Zhang ZY, et al: Integrated analysis of long non-coding RNA competing interactions reveals the potential role in progression of human gastric cancer. Int $\mathrm{J}$ Oncol 48: 1965-1976, 2016

40. He JH, Han ZP, Liu JM, Zhou JB, Zou MX, Lv YB, Li YG and Cao MR: Overexpression of long non-coding RNA MEG3 inhibits proliferation of hepatocellular carcinoma Huh7 cells via negative modulation of miRNA-664. J Cell Biochem 118 3713-3721, 2015.

41. Tong GF, Qin N, Sun LW and Xu XL: Long Noncoding RNA MEG3 suppresses glioma cell proliferation, migration and invasion by acting as competing endogenous RNA of MiR-19a. Oncol Res 25: 1471-1478, 2017

42. Liao M, Li B, Zhang S, Liu Q, Liao W, Xie W and Zhang Y: Relationship between LINC00341 expression and cancer prognosis. Oncotarget 8: 15283-15293, 2017.

43. Bozgeyik E, Igci YZ, Jacksi MF, Arman K, Gurses SA, Bozgeyik I, Pala E, Yumrutas O, Temiz E and Igci M: A novel variable exonic region and differential expression of LINC00663 non-coding RNA in various cancer cell lines and normal human tissue samples. Tumor Biol 37: 8791-8798, 2016.

44. Liu F, Yuan JH, Huang JF, Yang F, Wang TT, Ma JZ, Zhang L, Zhou CC, Wang F, Yu J, et al: Long noncoding RNA FTX inhibits hepatocellular carcinoma proliferation and metastasis by binding MCM2 and miR-374a. Oncogene 35: 5422-5434, 2016.

45. Montpetit A, Larose J, Boily G, Langlois S, Trudel N and Sinnett D: Mutational and expression analysis of the chromosome $12 \mathrm{p}$ candidate tumor suppressor genes in pre-B acute lymphoblastic leukemia. Leukemia 18: 1499-1504, 2004.

46. Tian Z, Wen S, Zhang Y, Shi X, Zhu Y, Xu Y, Lv H and Wang G: Identification of dysregulated long non-coding RNAs/microRNAs/mRNAs in TNM I stage lung adenocarcinoma. Oncotarget 8: 51703-51718, 2017. 